\title{
A Design Study to Improve User Experience of a Procedure Booking Software in Healthcare
}

\author{
Hanaa Abdulkareem Alzahrani ${ }^{1}$, Reem Abdulaziz Alnanih ${ }^{2}$ \\ Computer Science Department, Faculty of Computing and Information Technology \\ King Abdulaziz University, Jeddah, Saudi Arabia
}

\begin{abstract}
In the era of technology-driven healthcare delivery and the proliferation of e-health systems, procedure booking software is becoming common. Procedure booking software (PBS) affects healthcare delivery by improving health care efficiency and outcomes, while cutting costs. Therefore, poor software design for PBS, especially if it is designed for important and critical appointments such as cardiac catheterization operations, creates stress for physicians and may result in their rejection of this technology. Moreover, if the system design forces them to spend more time documenting health information, physicians would then tend to prefer face-to-face interaction with patients. Software with poor usability increases the workload of physicians thus reducing system efficiency. So designing a useful and effective web user interface for such software is an essential requirement for health websites. The aim of this paper is to design and develop a PBS as a case study using the health systems design (HSD) tool. HSD is a validated design tool for creating PBS based on physician behavior and persona. The applicability of a PBS design is explored by physicians evaluated. The PBS design was evaluated in terms of objective and subjective characteristics and user experience attributes. Test participants were divided into two groups: specialists and fellows. The results show that there was no significant difference between participants in either group. All were able to complete the tasks successfully with a minimum amount of time, clicks, and errors indicating that the effectiveness, efficiency and cognitive load were similar for all participants. User satisfaction yielded a score of 86 on the System Usability Scale (SUS), putting it in the A Grade. Also, user experience attributes demonstrated that participants were satisfied using the proposed design system.
\end{abstract}

Keywords-Procedure booking software; health systems design tool; cardiac catheterization; user experience; usability evaluation; system usability scale

\section{INTRODUCTION}

In the emerging era of technologically advanced health care and the proliferation of e-health systems, effective procedure booking software (PBS) is imperative [1]. PBS impacts healthcare delivery by improving health-care efficiency, reducing costs and improving health findings [2]. Designing a useful and effective web user interface (UI) for such software is an essential requirement for health websites. Even a minor change in a UI could lead to usability problems. For example, changing the background or foreground color of buttons could create difficulties for physicians. One of the reasons physicians are spending more time documenting patient health information is poor UI design for PBS which could lead them to reject the technology and prefer face-to-face interactions with patients [3].
Using a system for the first time, especially an outdated system, can be challenging. Unfortunately, it can be very difficult to replace or upgrade specific-purpose systems that require retraining physicians and integrating it with other services. Therefore, often new physicians asked to learn outdated systems that aligned with UI difficult to use.

The UI should be designed based on proven design principles, such as consistency between pages, ease of navigation, and also be eye-catching and user-friendly. All these characteristics are included in the usability definition. Usability refers to the degree to which a specific user often uses a product to attain specific aims with effectiveness, efficiency and satisfaction in a specific use context [4].

In the pediatric cardiology department at King Abdulaziz University Hospital (KAUH), the booking for cardiac catheterization is paper-based and can only be viewed and modified by visiting the clinic. There is no communication system between the cardiologist, the calendar and the hospital information data. As a result, cancellation or adjustment is likely to cause confusion in the cardiac catheterization team and even loss of bed appointments. Taking healthcare user experience (UX) into account in the creation of software systems can provide substantial benefits for the creation of successful healthcare systems, and also improve user overall well-being. However, designers must be familiar with medical expressions and understand human cognition [5].

The importance of this research is to propose a solution to the problem of health systems that are designed without taking into account the users requirements, preferences and goals in terms of speed and ease of use. The emergence of a UX that focuses on user requirements as the basis for designing UI, is the ideal solution for this problem.

The purpose of this study is to design an adaptable UI for PBS based on healthcare UX using the proposed health system design (HSD) tool. HSD is a tool-based website, whose design is based on physician persona and behavior. This tool maps healthcare personae from real life to the design space for healthcare UI systems, especially for PBS. The proposed PBS UI is evaluated and validated as proof of concept for the healthcare domain based on physician persona and behavior. This will assist the software designer to optimize UX and create persuasive software by understanding the type of behavior that needs to be considered when designing a UI [6].

The research question address in this study is, how can special software be designed for physicians? And how can its 
design be validated to measure physician work quality who lack a fundamental interest in technology?

The objectives of this research are as follow:

1) To create a PBS using the HSD tool (Sections III and IV).

2) To validate the PBS design and measure the quality of physician work as follows:

a) Measure objective attributes in terms of effectiveness, efficiency, and cognitive load.

b) Measure subjective characteristics in terms of the System Usability Scale (SUS).

c) Measure UX attributes in terms of completing the task the task's success and errors per task.

The rest of the paper is organized as follows: Section II presents the literature review. Section III describes the HSD tool. In Section IV details the PBS method and materials process. In section $\mathrm{V}$ the experimental design and evaluation are discussed and the results are illustrated in section VI. Finally, the conclusion and future of the work are presented in section VII.

\section{LITERATURE REVIEW}

In this section, the related literature is explored from two main streams, booking systems and usability evaluation.

\section{A. Booking Systems}

Web-based booking systems are important for reducing errors that can arise using paper-based systems. Researchers Zhao et al. searched PubMed seeking to identify advantages and barriers to implementation of web-based medical scheduling [7]. A total of 36 articles discussing 21 web-based appointment systems were selected. The results of this review suggests there are advantages to a variety of patient outcomes from web-based scheduling such as reducing no-show rates, waiting time, and staff work, while improving satisfaction. Otherwise, as barriers, patient reluctance to adopt web-based appointment scheduling is mainly affected by past experience with computer technology and the Internet. Primary and specialty clinics use appointment scheduling systems to manage access to service providers, and hospitals can also use an appointment system to plan elective surgery.

Gupta \& Denton described the most common healthcare delivery systems paying particular attention to the many factors that make appointment arrangements more challenging [8]. Factors including variability of arrival and service times, patient and provider preferences, available information technology, and the scheduling staff's level of experience [8]. In addition, the key bottleneck was determined to lie in the application of Industrial Engineering and Operations Research (IE/OR) technology. They provided the latest technology roadmap in the design of appointment management systems and identified future opportunities for novel applications of IE/OR model [8].

In Taiwan, Yang et al. revealed that more than half of hospitals have public online booking systems. However, they note that most systems perform only the registration function and rarely seize the opportunity to collect other information, such as related medical history or reasons for consultation [9]. They indicated further efforts should be made to strengthen the functions of these online booking systems in order to improve the efficiency of consultation. Some methods for information extraction and retrieval of unstructured medical records are needed to improve the efficiency of the appointment process.

Gamma et al. regard design patterns as a general solution to the recurring problems in software development, and therefore provides basic support to deal with these problems [10]. Appling the patterns of human-computer interaction provides basic design support and enhances development of the UI. The UI development model remains an important key theme. However, further research is needed to optimize its application.

Sinnig et al. applied the pattern concept to the analysis design of UI for online booking applications [11]. The application was tested with a usability evaluation to discover any other usability issues, thus optimizing its use. Their paper constitutes a step forward to integrating UI design patterns into the software development process.

According to Murray et al., the measurement of the performance of health systems relates goal attainment to the resources available [12]. They show that variety in performance is a function of how a health system organizes four key functions: stewardship, financing, service supply, and resource generation [12]. By investigating these four functions and how they are combined, they show it is possible to observe major policy challenges and understand the proximate determinants of health system performance.

The difference between the proposed approach and today's prevailing systems is: the proposed approach is based on user personas that plays an important role in the design orientation. Also, the proposed approach includes user's behavior. By considering persona and behavior that helps to understand the mental language of the target users and makes the whole experience in a systematic way quite humane.

\section{B. Usability Evaluation}

In 1998, the International Standards Organization released an original document containing requirements that describe the extent to which specific types of users can use a system to achieve specific goals of effectiveness, efficiency and satisfaction, as shown below [13]:

- Effectiveness: The completeness and accuracy in achieving a specific goal.

- Efficiency: Resources are used to improve the accuracy and completeness of users in achieving their goals.

- Satisfaction: Users don't feel any discomfort and have a positive attitude when using this product.

DeLone and Ephraim [14] described success terms of an information system (IS) defined as "a purposeful entity composed of interdependent computer-based technology and human components unified by design to accomplish one or more objectives." Interface satisfaction is defined as a dimension that captures the user's general satisfaction with the interface in terms of presentation, format, usability, and efficiency [14]. Decision support satisfaction is the level of 
satisfaction a user has with the help provided by the IS tool in planning or controlling a business process. Operational efficiency focuses on improvement in internal customer performance and is estimated with respect to flexibility, productivity, consistency, and process duration. Quality of work-life satisfaction is the level of user satisfaction as a result of the impact of the IS on their emotions, physical requirements, personal goals, and psychological states [14]. Finally, task support satisfaction is "the level of user satisfaction from the help provided by the IS tool toward the goal of achieving job and task requirements.

J. Rinder in her dissertation examined literature investigating website usability testing [15]. She reviewed 31 peer-reviewed articles, conference proceedings, and books identifying 10 usability testing categories, across a variety of testing methods, she noted that the most frequently listed categories addressed (a) navigation, (b) search features, and (c) content.

Usability evaluation has become a very important issue for websites since usability strongly affects users. Shasha \& Weideman undertook a usability study of Cape Town hotel reservation systems [16]. Employing usability testing as a research instrument to evaluate the system, they found that more than $52 \%$ of participants indicated key factors were content simplicity and an easy-to-understand booking system. $18 \%$ felt the websites were confusing, while $12 \%$ found the booking process frustrating. Their results provide a clear understanding how website usability affects user satisfaction.

A number of international standards on usability are available, but rarely used for useful usability evaluation. Hussain et al. used standard ISO 9241-11 to evaluate a webbased health awareness portal within the smartphone mobile context [17]. Their results uncovered some usability issues as well as confirming that the web-based awareness portal is relatively usable on smartphone devices within components defined in the models.

Gustafsson discussed how to design a UI for booking sunbeds to be both efficient and effective, while meeting the needs of first-time users [18]. Two personas were created to facilitate design of initial paper prototypes using user estimates. Then they iterated the design of the paper prototype and created a high-fidelity prototype using Adobe XD, which was evaluated using a task-based usability test. The prototype turned out to be very effective with additional qualitative data from participants helping to create an interesting experience and a system easy to learn and use.

Bangor et al. evaluated the system usability scale (SUS) from information collected on various systems during the various stages of the development life cycle over a 10-year period [19]. The SUS reflects a strong demand in the usability community for tools that can swiftly and efficiently collect users' subjective evaluations of usability. The information gathered in their study demonstrates that SUS can meet this need. Their analysis of SUS scores showed that for usability experts SUS is an exceptionally powerful and multifaceted tool.
Sauro conducted a five-year three-part study analyzing the experience of 4,000 users on more than 100 websites, thereby generating an eight-item website standardized UX percentile questionnaire featuring four factors: usability, trust, appearance and loyalty [20]. He concludes that questionnaires create reliable scores in benchmark websites, while standardized scores help designers to understand how the website scores compare with other scores in the database.

Feedback from developers, managers, and users is needed to optimize a system. In addition to basic software qualities, usability and UX are important attributes to improve. Usability is well known and can be tested, for example, by usability testing or expert reviews. On the other hand, UX describes the overall impact of the system on the end user before, during and after use.

Rauschenberger et al. introduced a tool that can easily evaluate the UX [21]. The tool is existing in multiple languages. They showed how to use the tool for continuous UX evaluation. Their work involved a validation study analyzing the Spanish version of the UX questionnaire, which they show can quickly evaluate the UX of interactive systems. Their tool measures usability aspects (such as efficiency, perspicuity, and dependability) and UX aspects. As the User Experience Questionnaire (UEQ) has a semantically different form, it is important that participants can use their natural language to rate products.

According to Kaur et al., the most important aspect of regulating the value of a website is usability [22]. Website designers need to understand the usability level of their website. Measurement techniques can be used to improve the usability level of a website. To determine usability level, they used two automated tools: a site analyzer which calculates multiple parameters and the Qualidator tool to check usability, accessibility, search engine optimization and technical quality. They evaluated educational universities in Punjab and provided rankings based on some evaluation criteria.

Based on the literature review the authors conclude the following:

1) There are many advantages and challenges to using PBS [7- 12].

2) There are different dimensions through which the usability of websites can be evaluated. The SUS is the most reliable option, even with a small number of participants, because of its reliability and low cost [15-22].

\section{THE HSD TOOL}

The HSD tool is a dynamic website based on physicians' persona. This tool provides various objects to designers of the health website. Each user has a dashboard that contains a set of different templates, or one can start a project from scratch. The tool provides the technologies and instruments used in the project in workspace panels such as page layout, adding an object (components, layouts, media, typography, buttons, and forms) and adding new pages. The user also has various options such as: change the setting, preview site and save code. The tool also contains code editors for maximum control. The HSD website implementation is based on the bootstrap 
framework. The programming languages used in the HSD website are HTML, CSS, SCSS, JavaScript, jQuery.

The benefits of this design tool include making it possible to create an early health site UI prototype which addresses user requirements and avoids many of the usual difficulties. Based on this tool, the authors designed a UI for a PBS prototype and evaluated its usability creating a system suited to the personae of healthcare providers that is effective, efficient, and userfriendly while also reducing stress levels and improving time management.

\section{Procedure Booking Software (PBS) MATERIALS AND METHODS}

In this section, the procedure for designing the PBS is described as follows:

- Define the design requirements: follow the manual PBS used by the pediatric cardiologist at (KAUH), Jeddah, Saudi Arabia. Define persona that characterize physician behavior and the scenarios used in real life. [5].

- Design and develop an HSD tool, and validate this tool by measuring usability and UX.

- Based on the validation, the PBS was designed and evaluated (see section V).

After designing the HSD tool and prior to using it for designing the PBS UI empirical research was conducted to gather information from six physicians, three specialists and three fellows, in the pediatric cardiology department at (KAUH). Specialists use the manual PBS to book patients and work under the fellows, the experts in the field, who perform the surgeries. A questionnaire was designed to collect important information from two areas: general information and specific questions related to the participant's experience using technology devices. For example: Do they think that using electronic healthcare booking is easier than paper records? Do they prefer to manage the booking procedure electronically? Do they feel comfortable using an electronic healthcare booking system? Do they require knowledge about how to use the electronic healthcare booking system? This step helped to reveal any assumptions about user knowledge and experience and created a starting point for decomposing any stereotypes. All the response results were collected and expressed as a percentage as follows:

- $71.4 \%$ of the responses were from the age group 30 to 39 years.

- $86 \%$ of the responses have a medium level of experience in using technology devices.

- All the responses their specialty was pediatric cardiology, $50 \%$ specialist and $50 \%$ fellow.

- $100 \%$ of the responses prefer to manage the booking procedure electronically.
- $57 \%$ of the responses used a paper-based booking procedure method and $43 \%$ a computer-based method.

- $86 \%$ of the responses believe using electronic healthcare booking is easier than paper records.

- $86 \%$ of the responses have the required knowledge to use the electronic healthcare booking system.

- $86 \%$ of the responses feel comfortable using the electronic healthcare booking system.

Based on the above results, the researchers decided to implement the PBS based HSD tool designed to suit the physicians' personalities and meet their requirements.

Establishing a PBS means designing a system that mainly deals with the process of booking operations. Operation room reservations are linked to the system so a physician can make reservations from his private clinic through the system. The method revolves around the physician's ability to enter the electronic system from any computer, mobile phone or tablet to enter the patient's data. Then the physician chooses the day of the operation, expected duration and type, either as a one-day surgery or hypnotic session. After this, the physician schedules the process electronically and approves it. The system is characterized by several features as follows:

- Friendly: It easily and accurately deals with the information.

- Arrangement: If the operation is canceled by the physician, the canceled day will be automatically carried over to the next available operation day.

- Notification: The patient is notified of the new appointment by letter.

- Reminder: Reminders are sent to the physician and the patient about the date of the operation, with the attendance confirmed by the patient, or the reservation is canceled, making it available to another person.

- Availability: The physician is able to enter the system and see all the private information, the date of the surgery, and all the observations of the operation.

- In addition, there is a special schedule for anesthesiologists, their names, hours of operation, and numbers so they are notified by email.

\section{A. Tools and Technologies}

Libraries and programming languages used for the PBS website are the same as for the HSD tool. The framework consists of HTML, CSS, SCSS, JavaScript, jQuery, and the bootstrap framework [23].

\section{B. Architecture and Implementation}

Fig. 1 illustrates the architecture applied to PBS website. 


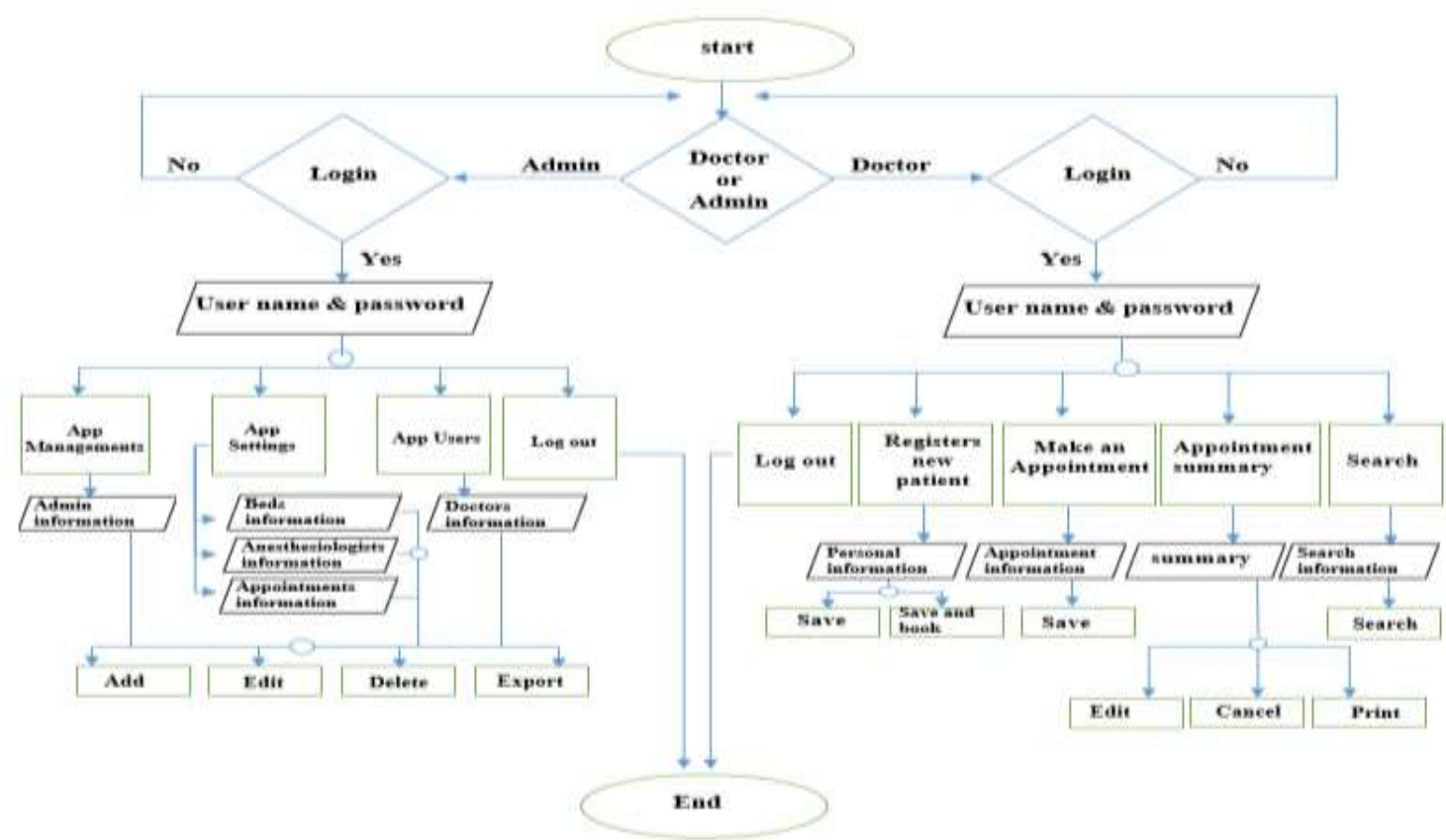

Fig. 1. Architecture of the Procedure Booking Software (PBS) Website.

\section{Prototype}

The majority of studies involving prototypes discuss the fidelity aspect of prototypes [24]. The concept of fidelity denotes the similarity between a prototype and the final product. Based on the degree of refinement, prototypes are categorized as low-fidelity or high-fidelity (hi-fi) [25]. The final hi-fi prototype was designed based on the outcomes from the low-fidelity early version, and the personae. The set of features provided by PBS allows users to register a new patient, book an appointment, display a summary of the appointment, and search for patient information throughout a website [26]. Two sections are harmonious all around the UI: menu section on the left bar, and the action section (dashboard) on the right bar. The site is divided into two basic pages. The user page presents materials for booking procedures. Here the user can register new patients and book appointments. The admin page is used to modify and control input and relevant processes and communicate and exchange with the user page (Fig. 2). During the design process, the website went through various revisions considering different parameters before reaching the final version.

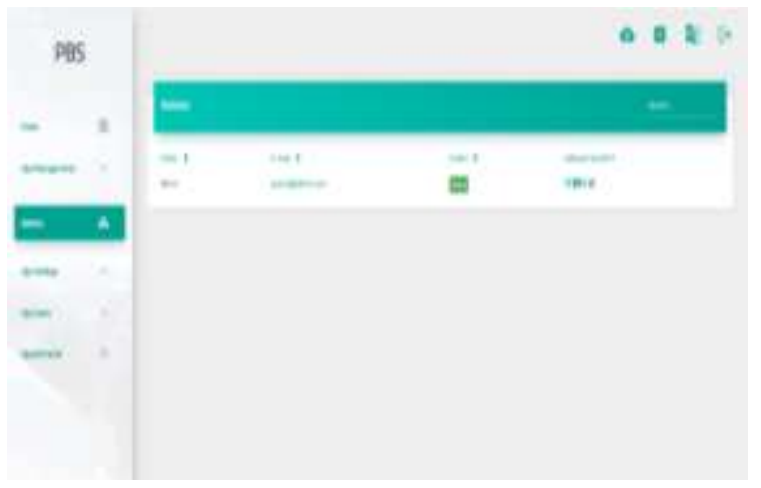

Fig. 2. The Admin main Page.

\section{EXPERIMENTAL DESIGN AND EVALUATION FOR THE PBS}

The experimental evaluation presented in this section involves the usability testing of PBS. Before conducting the testing, the following key issues require consideration for the information-gathering to be fruitful [27]:

\section{A. Defining the Goal}

The goal of this study is to design a PBS based on the HSD tool to improve effectiveness, efficiency, and physician satisfaction —as usability definition in ISO 9241-11 [28].

\section{B. Pilot Test}

A pilot study is a preliminary run of the principle study. The point is to ensure that the proposed strategy is practical before setting out on the real examination. The equipment and instructions that should be used were prepared.

The proposed PBS was tested first with one expert designer and one expert physician. Both were asked to complete the list of tasks in Table II to ensure the design is clear and works well. The researchers received feedback before conducting the test. Several adjustments were made before conducting the pilot test and after, with the clarification of the actions. Table I contains sample of Pilot test 1 with several changes: (added the necessary items, changed some terms name, added searching). Pilot test 2 with several changes: (add content to the home page, change the format of the entered lists, resize all buttons). Pilot test 3 with several changes: (changed some terms, make the date a full (day, month and year), change some terms, add an advanced search).

\section{Defining the Participants}

Participant samples were comprised of six physicians selected at random for the study belonging to the department of paediatric cardiology at KAUH. Three participants were 
fellows (Novice) and three specialists (Expert) in using the existing booking method. All the participants were asked to perform the same list of tasks individually, and then answer the post-test questionnaire. The attributes collected: the time spent to complete each task, the number of clicks, and the number of errors for each task. All the attributes were tracked using video recordings. After completing the tasks, the participants answered SUS questionnaire [29], and were requested to offer inputs related to their experience on a short note.

TABLE I. The PiLot Test

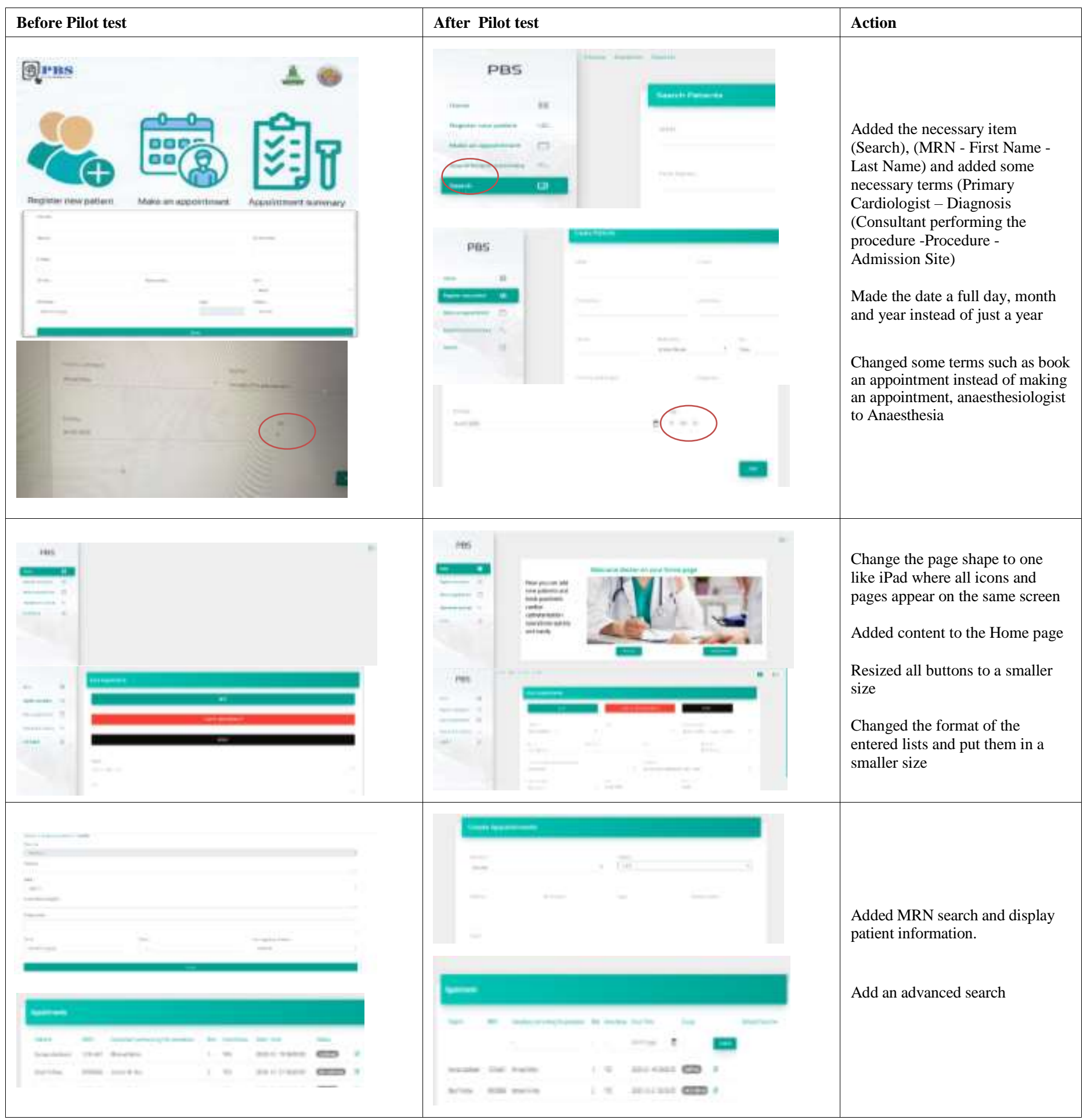


To determine the effectiveness of the proposed tool, the two groups were defined as follows:

- Specialists group (Expert): The least experienced in the field but the most familiar with electronic systems.

- Fellow group (Novice): The most experienced in the field but the least familiar with electronic systems.

The test was conducted in the summer 2020. Due to the outbreak of COVID 19, the evaluation was conducted in online environment. The researchers contacted the participants first by sending the consent form to obtain their approval and explain the reason for conducting the study. All the participants received the same set of instructions. For example: 1) Participants were asked to open the screen recorder to record each session before starting to perform the test. 2) Participants were asked to fill out the SUS. Then, asked to perform a list of tasks (Table II) on the PBS link on the web-based system and download the recording screen to record their performance, then return it to the researchers by email.

The participants were asked to perform the tasks without explanation of the system. All the participants received four tasks to complete reflecting different functionality as follows (Table II):

TABLE II. FOUR TASKS TO EXAMINING THE PBS SYSTEM

\begin{tabular}{|c|c|c|}
\hline & Task & Description \\
\hline 1 & $\begin{array}{l}\text { Register a new } \\
\text { patient. }\end{array}$ & $\begin{array}{l}\text { Input MRN (any } 7 \text { number), E-Mail (any email), } \\
\text { First Name (any name), Family name (any name), } \\
\text { Tel No (any mobile number), Nationality (Saudi } \\
\text { Arabia), Sex (male or female), Primary } \\
\text { Cardiologist (Khadijah Maghrabi), Diagnosis } \\
\text { (Double outlet right ventricle), Birthday (any } \\
\text { date) and click SAVE. }\end{array}$ \\
\hline 2 & $\begin{array}{l}\text { Book an } \\
\text { appointment }\end{array}$ & $\begin{array}{l}\text { Choose MRN that you registered then input Bed } \\
\text { (1 or 2), Anesthesia (YES | NO), Consultant } \\
\text { performing the procedure (Jameel Al-Ata), } \\
\text { Procedure (Closure of PDA), Admission Site } \\
\text { (Pediatric ward), Date (any date), Time (any time) } \\
\text { and click SAVE. }\end{array}$ \\
\hline 3 & $\begin{array}{l}\text { Display a summary } \\
\text { of the appointment. }\end{array}$ & Display all appointments. \\
\hline 4 & $\begin{array}{l}\text { Search about the } \\
\text { patient }\end{array}$ & $\begin{array}{l}\text { Search about the patient that you enter by his } \\
\text { MRN or by his name. }\end{array}$ \\
\hline
\end{tabular}

\section{Triangulation Role}

The triangulation of data is drawn from a different sampling technique (observation recorded video and questionnaire) that was used to collect and interpret the data. The authors chose video recording because video has the advantage of capturing both visual and audio data. Also, results from a video session can be taken away and analyzed. Participants had to use their own desktop computers supported with Google Chrome to test the PBS.

\section{RESULTS}

This section first provides the results of the usability study in terms of objective characteristics. Then, the subjective characteristics in terms of SUS results and finally, the UX attribute measures.

\section{A. The Usability Study Result}

The usability testing was conducted to measure the effectiveness, efficiency, and cognitive load.

To make the test completely scientific, the authors set two hypotheses and performed a T-test to determine whether there was a significant difference between the two groups [30].

- Effectiveness:

The effectiveness was measured using the total min correct clicks divided by the sum of correct clicks and incorrect clicks per task.

Effectiveness $=\frac{\text { Min correct clicks }}{\text { correct clicks }+ \text { incorrect clicks }}$

The assumption was relied on the data to support or reject the hypotheses in effectiveness characteristics based on the following hypotheses:

- Hypothesis 01: There is no significant difference between the effectiveness of the specialists group and fellow group.

- Hypothesis 1: There is a significant difference between the effectiveness of the specialists group and the fellow group.

Based on the average result for all tasks, the P-value was $(0.16)$ greater than the value of alpha $(0.05)$, which means that the hypothesis 01 is not refuted. Fig. 3 shows that the fellow group is better at completing all tasks than the specialist group, except task4.

\section{- Efficiency:}

The efficiency was measured using the effectiveness divided by the total time spent per task.

Efficiency $=\frac{\text { Effectiveness }}{\text { Time }}$

The assumption was relied on the data to support or reject the hypotheses in efficiency characteristics measured based on the following hypotheses:

- Hypothesis 02: "There is no significant difference between the efficiency of the specialists group and fellow group."

- Hypothesis 2: There is a significant difference between the efficiency of the specialists group and the fellow group.

Based on the average result for all tasks, the P-value (0.10) is greater than the value of alpha $(0.05)$. The result conclude that there is no significant difference in efficiency values between the specialists group and the fellow group. Fig. 4 shows that the fellow group had higher efficiency compared to the specialist group in completing the tasks. This indicates the fellow group performed better than the specialist group. 


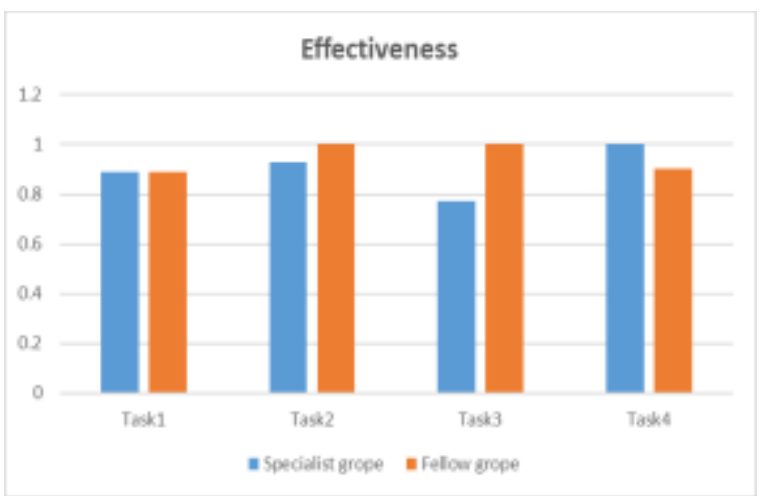

Fig. 3. Calculations for Effectiveness.

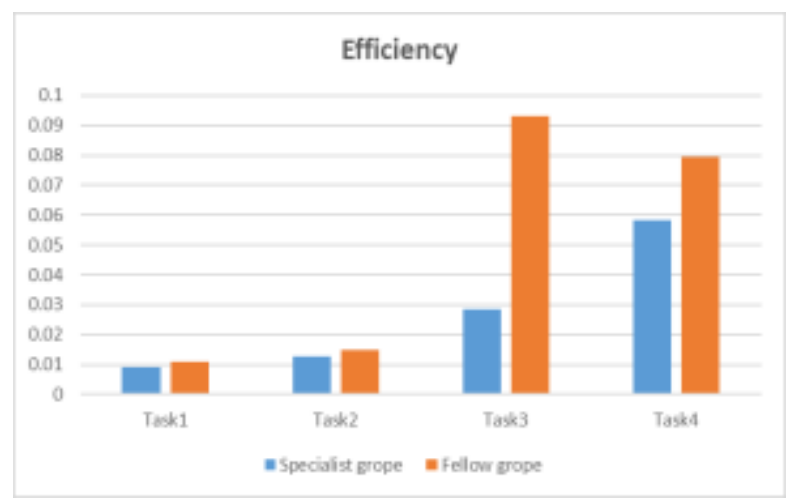

Fig. 4. Calculations for Efficiency.

\section{- Cognitive load:}

To accomplish a 'task', a user may have to switch from one screen to another. This will depend on the complexity of tasks, screen size, or the way the designer has filled information on various screens [31]. It was measured by:

\section{Cognitive load $=\frac{\text { Pages }^{\prime} \text { view }}{\text { correct clicks }+ \text { incorrect clicks }}$}

The assumption was relied on the data to support or reject the claims in cognitive load characteristics measured based on the following hypotheses:

- Hypothesis 03: "There is no significant difference between the efficiency of the specialists group and fellow group."

- Hypothesis 3: There is a significant difference between the efficiency of the specialists group and the fellow group.

Based on the average result for all the tasks, the P-value obtained (0.21) is greater than the value of alpha (0.05), which means that cognitive load hypothesis 03 is not rejected.

Fig. 5 shows that the fellow group had equal or higher cognitive load than the specialist group in completing the tasks except for task 4 which means the specialist group was better in cognitive load than the fellow group. This result explains the specialist's familiarity with using the technology since their cognitive load was better than the fellow in most of the tasks except Task 4.

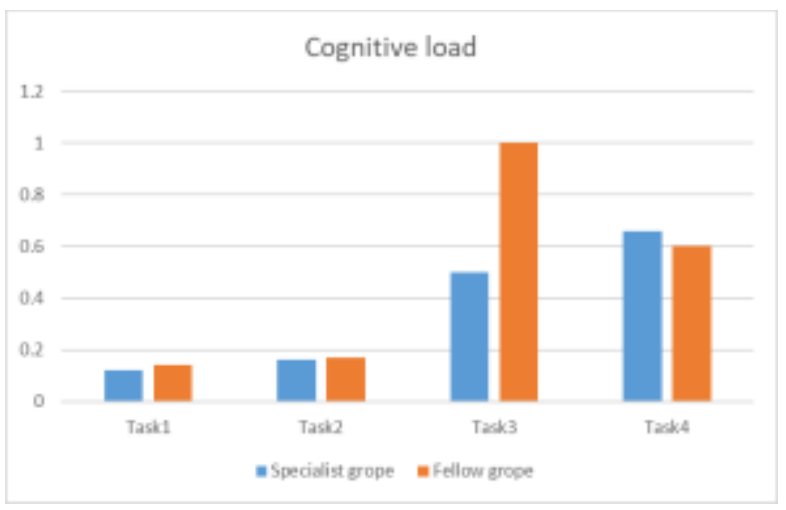

Fig. 5. Calculations for Cognitive Load.

In terms of effectiveness and efficiency, all participants in the two groups were able to successfully complete each task, which means that they understand the task to be performed using the system and have the ability to perform that task.

Overall, there is no significant difference between the two groups for either of the tasks. However, there is a slight difference in the number of clicks and the time spent between the participants, as the fellows were quicker than the specialists in terms of time and required less clicks. See Fig. 6 (The average number of clicks required per task), and Fig. 7 (The average completion time per task).

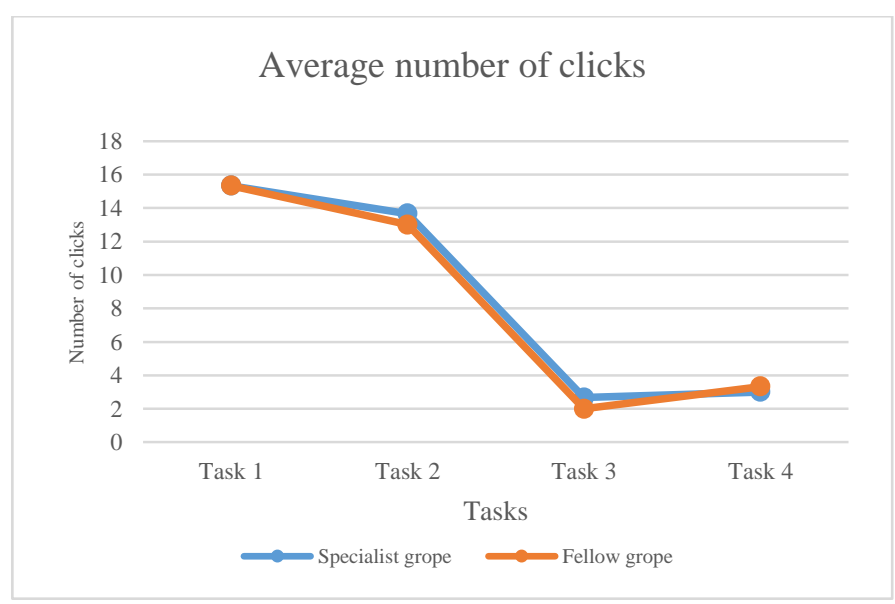

Fig. 6. Total Number of Clicks.

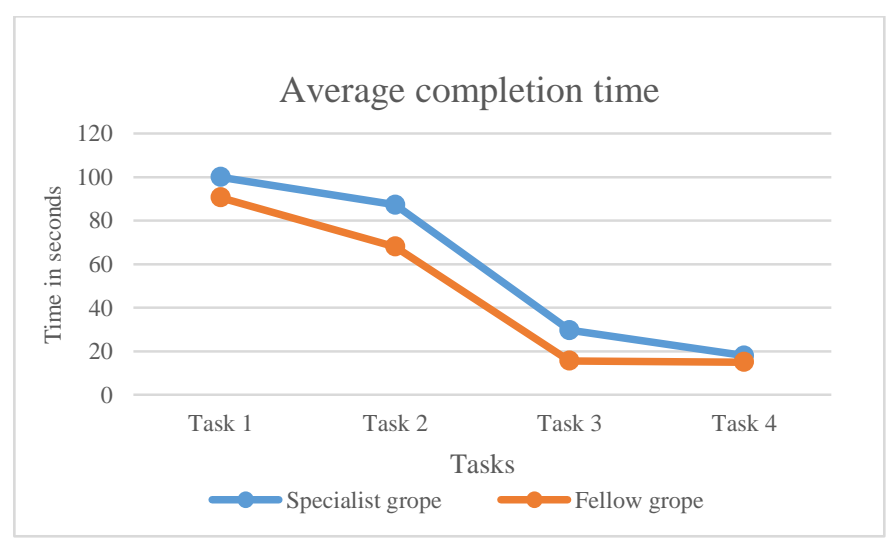

Fig. 7. Total Time to Complete the Tasks. 


\section{B. System Usability Scale Result}

The questionnaire was followed up with a short Google survey. For all participants in both groups the lowest SUS score landed on 65 while the highest was 100 . The average SUS score was 86 setting it in the A Grade.

The SUS questionnaire includes the following criteria:

1) I prefer to use the PBS regularly.

2) I found the PBS unnecessarily sophisticated.

3) I found the PBS was easy to use.

4) I would need the help of a technical person to use the PBS.

5) I found the different functions in the PBS integrated well.

6) I found there was a lot of discrepancy in the PBS.

7) I can claim that most people would learn to use the PBS very quickly.

8) I found the PBS very burdensome to use.

9) I felt very confident using the PBS. PBS.

10)I need to learn many items before working with the

Comments mentioned by most of the physicians are as follow:

- "User-friendly"

- "Easy to learn and organized system"

- "This system is good for its ability to save booking data from being lost, and it is good for easy search /recall saving a lot of time needed to look for a booked patient. Thank you for developing this system to help us"

- "Its efficient, user friendly and saves time"

- "So easy and comfortable"

- "Maybe we need additional digits like additional notes"

\section{User Experience Attributes Result}

The PBS was evaluated by measuring the UX attributes in terms of time -success - errors [32]. The results are as follows:

- Time to complete the tasks:

Fig. 8 shows that all participants in both groups completed all tasks in the best time. The fellow group was faster than the specialists group in all tasks.

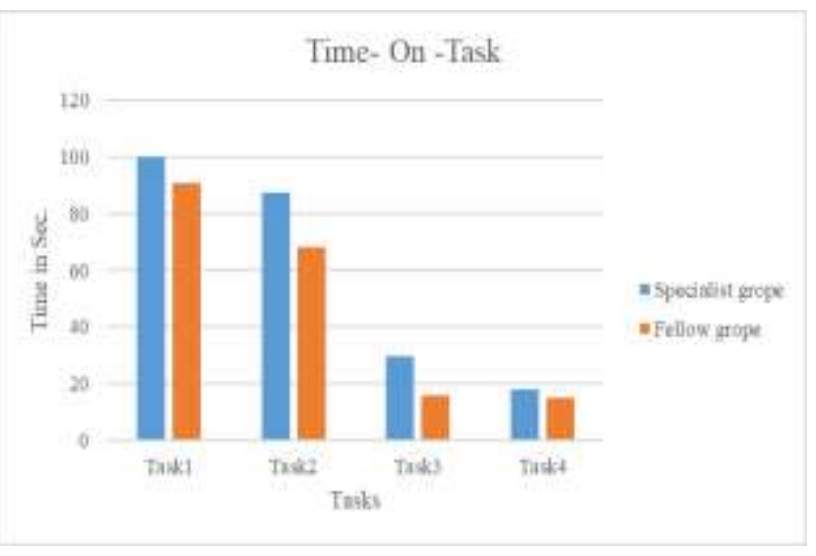

Fig. 8. Total Time to Complete the Tasks.
- Successful tasks percentage:

Fig. 9 shows that all participants in both groups had a success rate of $100 \%$.

\section{Successful Tasks Percentage}

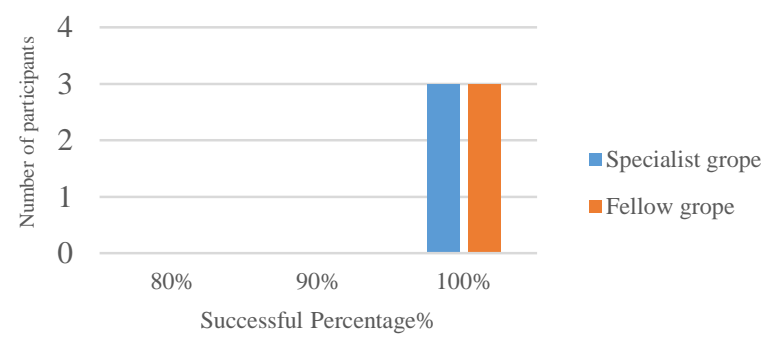

Fig. 9. Total Successful Tasks Percentage.

- Number of errors percentage:

Fig. 10 shows that participants in both groups had less than $10 \%$ error in completing all tasks. Just one specialist had errors close $20 \%$ in task 1 .

From the results on the usability study, SUS and UX attributes, the authors can confirm that the proposed PBS is user friendly, easy to use, easy to learn and satisfy the user with minimum effort and time. This is supported by the observation that most of the results of the fellow (novice user) is better or close to the specialist (expert users).

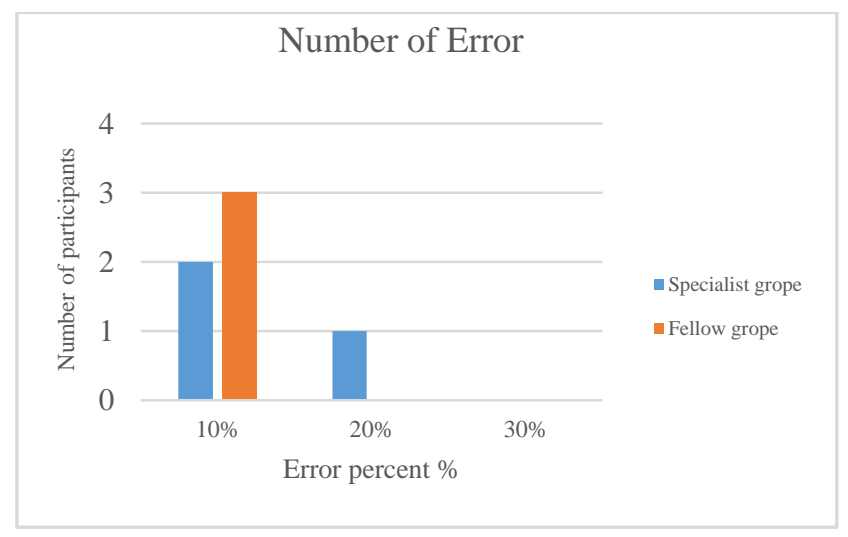

Fig. 10. Total Number of Errors.

\section{CONCLUSION AND FUTURE WORK}

This research aimed to design and develop a suitable PBS UI as a case study by using the HSD tool based on physicians' persona to prove the study design concept in the pediatric cardiology department KAUH. It was then measured the objective characteristics (effectiveness, efficiency, and cognitive load) and subjective features (satisfaction) by applying the SUS measurement. The results confirm that: there is no significant difference between specialists and fellow groups, which supports the study claim. Specifically, the fellow group achieved similar results to the specialists' group indicating the PBS was perceived as efficient, effective, and satisfactory when tested on six participants in a task-based usability test that obtained a SUS score of 86. 
UX attributes and user feedback confirmed that the test created a good experience that helped them perceive the system as efficient, user-friendly, and time saving.

In future work, the authors are looking to enhance the tool by generating the UI automatically based on the physicians' personality and expanding the persona, for example, adding a persona for nurses. Also, make it adapted for other health systems and testing with a larger sample.

\section{ACKNOWLEDGMENT}

The authors gratefully acknowledge the pediatric cardiology staff in the department of Cardiac Catheterization at KAUH.

\section{REFERENCES}

[1] Ellingsen G, Obstfelder A. Collective expectations-Individual action implementing electronic booking systems in Norwegian health care. International Journal of Medical Informatics. 2007 Jun 1;76:S104-12.

[2] Zhao P, Yoo I, Lavoie J, Lavoie BJ, Simoes E. Web-based medical appointment systems: a systematic review. Journal of medical Internet research. 2017;19(4):e134. R. Keefer, "Reducing Stress in Healthcare: Evidence from Using an Integration Design Model," pp. 705-709, 2017.

[3] M. Y. Ivory and M. A. Hearst, "Improving Web Site Design," no. April, 2002.C.

[4] International Organisation for Standardisation ISO 9241-11:1998 Guidance on usability.

[5] Alzahrani, Hanaa, and Reem Alnanih. "The Effect of User Experience on the Quality of User Interface Design in Healthcare." International Conference on Computing. Springer, Cham, 2019.

[6] B. Fogg, "The Behavior Grid," Proc. 4th Int. Conf. Persuas. Technol. Persuas. '09, p. 1, 2009.

[7] Zhao, Peng, et al. "Web-based medical appointment systems: a systematic review." Journal of medical Internet research 19.4 (2017): e134.

[8] Gupta D, Denton B. Appointment scheduling in health care: Challenges and opportunities. IIE transactions. $2008 \mathrm{Jul}$ 21;40(9):800-19.

[9] Yang PC, Chu FY, Liu HY, Shih MJ, Chen TJ, Chou LF, Hwang SJ. Features of online hospital appointment systems in Taiwan: a nationwide survey. International Journal of Environmental Research and Public Health. 2019 Jan;16(2):171.

[10] E. Gamma and R. Helm and R. Johnson and J. Vlissides, Design Patterns - Elements of Reusable Object-Oriented Software, AddisonWesley, 2002. 24th edition.

[11] D. Sinnig, A. Gaffar, D. Reichart, P. Forbrig and A. Seffah, Patterns in Model-Based Engineering, Proc. CADUI 2004.

[12] Murray, Christopher JL, and Julio Frenk. "A framework for assessing the performance of health systems." Bulletin of the world Health Organization 78 (2000): 717-731.

[13] Iso IS. 9241-11 (2018) Ergonomics of human-system interaction - part 11: usability: definitions and concepts. International Organization for Standardization. https://www. iso. org/obp/ui/\# iso: std: iso. 2018;9241(11).
[14] DeLone, William H., and Ephraim R. McLean. "Information systems success measurement." Foundations and Trends® in Information Systems 2.1 (2016): 1-116.

[15] Rinder, Julie. "The Importance of Website Usability Testing." (2012).

[16] Shasha,ZT and Weideman M. Usability measurement of web-based hotel reservation systems. Conference Proceedings of the 1 st International TESA Conference, September 2016, Cape Town, South Africa.

[17] Hussain, Azham, Emmanuel OC Mkpojiogu, and Zakaria Hussain. "Usability evaluation of a web-based health awareness portal on Smartphone devices using ISO 9241-11 model." Jurnal Teknologi 77.4 (2015): $1-5$.

[18] Gustafsson, Filip. "An Explorative Design Study of a Booking System: Evaluating the Usability and Experience of a User Interface for Novice Admin Users." (2019).

[19] Bangor A, Kortum PT, Miller JT. An empirical evaluation of the system usability scale. Intl. Journal of Human-Computer Interaction. $2008 \mathrm{Jul}$ 29;24(6):574-94.

[20] Sauro J. SUPR-Q: A comprehensive measure of the quality of the website user experience. Journal of usability studies. $2015 \mathrm{Feb} 1 ; 10(2)$.

[21] Rauschenberger, Maria, et al. "Efficient measurement of the user experience of interactive products. How to use the user experience questionnaire (UEQ). Example: Spanish language version." (2013).

[22] Kaur S, Kaur K, Kaur P. Analysis of website usability evaluation methods. In2016 3rd International Conference on Computing for Sustainable Global Development (INDIACom) 2016 Mar 16 (pp. 10431046). IEEE.

[23] Spurlock, Jake. Bootstrap: Responsive Web Development. O'Reilly Media, Inc.", 2013.

[24] Nissinen, Tuomas. "User experience prototyping-a literature review." University of Oulu, Oulu (2015).

[25] Coyette, Adrien, Suzanne Kieffer, and Jean Vanderdonckt. "Multifidelity prototyping of user interfaces." IFIP Conference on HumanComputer Interaction. Springer, Berlin, Heidelberg, 2007.

[26] Alzahrani, H., 2020. PBS Procedure Booking Software. [online] Booking-ps.com. Available at: 〈http://booking-ps.com/> [Accessed 13 October 2020]

[27] Sharp H and Preece J,, Interaction Design: Beyond Human-Computer Interaction, Wiley, p. 841, 2015.

[28] "ISO - ISO 9241-11:2018 - Ergonomics of human-system interaction Part 11: Usability: Definitions and concepts." [Online]. Available: https://www.iso.org/standard/63500.html. [Accessed: 11-Apr-2020].

[29] Peres, S. ., Peres, C., Pham, T., \& Phillips, R. (2013). Validation of the System Usability Scale (SUS): SUS in the wild. In Proceedings of the Human Factors and Ergonomics Society Annual Meeting (pp. 192-196). Santa Monica, CA: HFES.

[30] Talib MA, Alnanih R, Khelifi A. Application of quality in use model to assess the user experience of open source digital forensics tools. International Journal of Electronic Security and Digital Forensics. 2020;12(1):43-76.

[31] Reem Alnanih and Olga Ormandjieva and Radhakrishnan, T. (2013 C). A New Quality-in-Use Model for Mobile User Interfaces. Proceedings of the 23nd International Workshop on Software Measurement, IWSMMENSURA.

[32] Albert W, Tullis T. Measuring the user experience: collecting, analyzing, and presenting usability metrics. Newnes; 2013 May 23. 